\title{
Video Article \\ Sample Preparation Strategies for Mass Spectrometry Imaging of 3D Cell Culture Models
}

\author{
Dorothy R. Ahlf Wheatcraft ${ }^{1,2}$, Xin Liu ${ }^{1,2}$, Amanda B. Hummon ${ }^{1,2}$ \\ ${ }^{1}$ Department of Chemistry and Biochemistry, University of Notre Dame \\ ${ }^{2}$ Harper Cancer Research Institute, University of Notre Dame \\ Correspondence to: Amanda B. Hummon at ahummon@nd.edu
}

URL: https://www.jove.com/video/52313

DOI: doi: $10.3791 / 52313$

Keywords: Bioengineering, Issue 94, 3D cell culture, mass spectrometry, imaging, cell culture, sample preparation, spheroids

Date Published: $12 / 5 / 2014$

Citation: Ahlf Wheatcraft, D.R., Liu, X., Hummon, A.B. Sample Preparation Strategies for Mass Spectrometry Imaging of 3D Cell Culture Models. J. Vis. Exp. (94), e52313, doi:10.3791/52313 (2014).

\section{Abstract}

Three dimensional cell cultures are attractive models for biological research. They combine the flexibility and cost-effectiveness of cell culture with some of the spatial and molecular complexity of tissue. For example, many cell lines form 3D structures given appropriate in vitro conditions. Colon cancer cell lines form 3D cell culture spheroids, in vitro mimics of avascular tumor nodules. While immunohistochemistry and other classical imaging methods are popular for monitoring the distribution of specific analytes, mass spectrometric imaging examines the distribution of classes of molecules in an unbiased fashion. While MALDI mass spectrometric imaging was originally developed to interrogate samples obtained from humans or animal models, this report describes the analysis of in vitro three dimensional cell cultures, including improvements in sample preparation strategies. Herein is described methods for growth, harvesting, sectioning, washing, and analysis of 3D cell cultures via matrix-assisted laser desorption/ionization-mass spectrometry (MALDI-MS) imaging. Using colon carcinoma 3D cell cultures as a model system, this protocol demonstrates the ability to monitor analytes in an unbiased fashion across the 3D cell culture system with MALDI-MSI.

\section{Video Link}

The video component of this article can be found at https://www.jove.com/video/52313/

\section{Introduction}

Mass spectrometry imaging (MSI) is a powerful technique to examine the distribution of molecular species across samples of interest. Researchers have imaged biological samples from whole animals down to single cells with this technique. ${ }^{1-4}$ Using MSI hundreds of analytes may be imaged at a single time, rather than single-substrate techniques requiring the development of additional fluorophores for traditional confocal microscopy, or staining for histology. ${ }^{5-7}$ Additionally, MSI can be used in either a targeted or a discovery-based fashion, making it an attractive approach to look at the overlap between the distribution of chemical species. Presented within are methods for the growth, preparation, and matrix assisted laser desorption ionization/desorption (MALDI) imaging of small samples ( 1 mm in diameter) of three-dimensional multicellular cell cultures. ${ }^{8-11}$

3D cell cultures are of particular interest to the biological community as an intermediary between traditional monolayer culture and in vivo tumors. As the name implies, 3D cell cultures are heterogeneous cellular aggregates that contain many of the chemical gradients in the microenvironment observed in small avascular tumor nodules, making them a more realistic model system to the native tumor environment than monolayer culture ${ }^{10}$ Many human cell lines can be grown as 3D cultures, including cell lines derived from colon, breast, ovary, prostate, kidney and lung tissues. ${ }^{10}$ There are inherent challenges in imaging three dimensional cell cultures as they are smaller than many samples commonly used for mass spectrometry imaging and therefore require careful sample preparation. ${ }^{7}$ However, the advantages of these tractable model systems include lower cost and faster growth and analysis time compared to animal models. As a result, studies involving three dimensional cell cultures can be higher throughput than traditional animal studies. ${ }^{12-14}$

All of these advantages have resulted in increasing popularity for three dimensional cell cultures in biological research; there is a corresponding need to develop new analytical strategies to explore both the composition and spatial distribution of molecules in these systems. ${ }^{12,15-21}$ The goal of this manuscript is to describe the adaptation of mass spectrometry imaging methods and sample preparation to three dimensional cell cultures.

\section{3D Cell Culture and Preparation for Imaging}

1. Culture an appropriate cell line, i.e., HCT116 colon carcinoma cell line, in two dimensional, monolayer culture. 
2. Grow HCT 116 cells in a T-25 flask with McCoy's $5 \mathrm{~A}$ media $+10 \%$ fetal bovine serum in a $5 \% \mathrm{CO}_{2}$ incubator until $50-70 \%$ confluency, which generally takes a few days.

3. Release cells with $0.25 \%$ trypsin-EDTA solution and count a portion of the cells using a hemocytometer and trypan blue staining to check for cell density and viability.

4. After counting, dilute cells with complete medium to achieve an appropriate seeding density of $6,000 \mathrm{cells} / \mathrm{well}$, or $30,000 \mathrm{cells} / \mathrm{ml}$.

\section{Prepare Agarose Coated 96 Well Plates}

1. Add $0.19 \mathrm{~g}$ of agarose to $10 \mathrm{ml}$ of standard media in a $50 \mathrm{ml}$ conical tube $\left(1.5 \%\right.$ agarose solution in media) ${ }^{10}$. Ensure incorporation by gentle mixing. Autoclave the agarose in a water bath for $20 \mathrm{~min}$.

2. Using a multichannel pipette, aspirate $50 \mu \mathrm{l}$ of the agarose + media mixture into the 60 central wells of the flat bottomed 96 well plate culture plate. As the wells at peripheral edges of the plate have slightly higher evaporation rates, add $200 \mu \mathrm{l}$ x phosphate buffered saline (PBS) instead of the agarose to these edges.

3. Once the agarose mixture has been added to the inner wells, set the plate aside to cool to $\sim 37^{\circ} \mathrm{C}$ before the addition of the cells.

\section{Seed and Tend the Three-dimensional Culture}

NOTE: More or fewer cells may be seeded depending on the requirements of the culture involved, but it has been determined that these conditions result in 3D cell culture structures of approximately $1 \mathrm{~mm}$ in diameter after 10-14 days of culture (data not shown).

1. Add $200 \mu \mathrm{l}$ of the appropriate cell solution (diluted to contain $\sim 6,000$ cells) to the agarose-coated 96 well plate. Cover the plate and set in humidified incubator with $5 \% \mathrm{CO}_{2}$ at $37^{\circ} \mathrm{C}$ for cell growth.

2. Change cell media a minimum of every $48 \mathrm{hr}$, or when the media appears to be changing color.

1. Change media by carefully aspirating the old media from around the small $3 \mathrm{D}$ cell culture (visible to the naked eye by day 2 ) at the bottom of the agarose. Add $200 \mu \mathrm{l}$ of fresh media gently over the 3D cell culture.

2. (Optional step) During these media changes, add chemotherapeutics or other drugs for testing. Dilute the drug of choice to the fina concentration with complete media and add the requisite $200 \mu \mathrm{l}$ to each well.

3. Monitor 3D cell culture growth by optical microscope, until the 3D cell cultures are approximately $1 \mathrm{~mm}$ in diameter.

\section{Harvest the 3D Cell Cultures}

1. Use a $2 \mathrm{ml}$ serological pipette to gently remove the $3 \mathrm{D}$ cell culture from the agarose bed.

2. Wash the 3D cell cultures with PBS by gently pipetting them into approximately $1 \mathrm{ml} \mathrm{PBS}$ in a waiting 24 well plate.

3. Transfer harvested 3D cell cultures via serological pipet to centrifuge tubes for protein or metabolite extraction, or embed them in a cutting medium to assist in slicing for imaging applications.

\section{Embed the 3D Cell Cultures in Gelatin}

1. Prepare a solution of $\sim 175 \mathrm{mg} / \mathrm{ml}$ gelatin in high purity water ( $18 \mathrm{M} \Omega$ preferred). ${ }^{22,23}$ Mix the gelatin vigorously. Observe the solution becoming viscous and difficult to manipulate. Place the gelatin-containing conical tube in a water bath at $\sim 60{ }^{\circ} \mathrm{C}$ and warm until the solution becomes clear and easy to aspirate.

NOTE: The warm gelatin solution hardens rapidly as it cools, so perform all of the following steps rapidly before freezing.

2. After it is heated, take the gelatin immediately to the cell culture hood. Keep the tube with gelatin in a beaker with preheated water at about $60^{\circ} \mathrm{C}$ to avoid hardening of the gelatin solution.

3. Using a $2 \mathrm{ml}$ serological pipette, deposit $0.6 \mathrm{ml}$ of the warm gelatin solution into the well of a 24 flat-bottomed plate. This volume is sufficient to cover the bottom in a thin layer. Gently deposit the 3D cell cultures immediately on top of the gelatin layer using a different $2 \mathrm{ml}$ pipette to avoid rupturing the 3D structure.

NOTE: Care must be taken at this stage not to place PBS into the gelatin. If this happens, however, remove the PBS from the top of the gelatin using a $200 \mu \mathrm{l}$ pipette.

4. After the 3D cell cultures are placed on the surface of the gelatin layer, carefully pipette another $0.6 \mathrm{ml}$ of the warm gelatin mixture over the $3 \mathrm{D}$ cell cultures, so as to not disturb the position of the cultures. After the second layer is placed, freeze the gelatin-embedded 3D cell cultures at $-80^{\circ} \mathrm{C}$.

\section{Slice and Thaw Mount the Gelatin-embedded 3D Cell Cultures for Mass Spectrometric Imaging}

1. Remove the 24 well plate containing the $3 \mathrm{D}$ cell cultures from the freezer. Warm the bottom of the plate with the heat from your hand until a tweezer or scalpel will slide gently down the side of the well.

2. Smoothly slide the tweezer or scalpel, freeing the gelatin without thawing the center. Take a drop of water to the cryostat support and use this to adhere the gelatin disk to the support.

1. $3 \mathrm{D}$ cell cultures embedded in gelatin are most amenable to slicing at $-30{ }^{\circ} \mathrm{C}$. Clean the cryostat blade and glass with $70 \%$ ethanol before use. Use a different part of the blade or a new blade for each 3D cell culture-gelatin disc to prevent dulling of the blade.

3. Using the cryostat, gently face off the excess gelatin until the 3D cell cultures become visible.

4. At this point slowly slice the $3 \mathrm{D}$ cell cultures with a smooth motion in $12-16 \mu \mathrm{m}$ sections. 
NOTE: Critical factors to slicing include the distance of the glass to the blade, the blade angle, the temperature of the cryostat, and the angle of the disk on the support, so all of these must be checked to ensure consistent results.

5. Carefully thaw the slices and mount them on indium titanium oxide (ITO)-coated glass slides labeled appropriately and store it in a desiccator. Use the slices as quickly as possible for maximum quality.

\section{Matrix Application and Sample Preparation for MALDI Imaging}

1. Prior to MALDI imaging, prepare slices with the appropriate matrix.

1. For small molecule analysis, no washing steps are generally required. For intact protein detection, wash the slices in cold $100 \%$ acetone, Carnoy's fluid, or $70 \% / 95 \%$ isopropanol to remove small molecules such as lipids that could mask the signal. ${ }^{24-26}$

2. Gently wash for no more than $30 \mathrm{sec}$ at a time. Do not to disturb any slices.

2. Prepare matrix in a solution of organic solvent and water, with $0.1 \%$ TFA. For small molecule such as a-Cyano-4-hydroxycinnamic acid (CHCA), use $60 \% \mathrm{ACN}: 40 \% \mathrm{H}_{2} \mathrm{O}: 0.1 \%$ TFA $(10 \mathrm{mg} / \mathrm{ml})$. For protein detection such as sinapic acid $(30 \mathrm{mg} / \mathrm{ml})$, use the same solvent solution. For optimal solubility of the sinapic acid, dissolve first in acetonitrile before addition of the TFA: $\mathrm{H}_{2} \mathrm{O}$ solution. Other matrices may be used as appropriate.

NOTE: Matrix can be applied with several different methods, but it should be noted some matrixes have been found to co-crystalize with the gelatin, such as ferulic acid, rendering the sample unusable. Smaller crystals produce more consistent mass spectra, allowing for more molecular species to be detected and attributed to biological differences rather than matrix effects.

3. Apply matrix by handspotting method.

1. Use a fine-tipped syringe to apply $0.5 \mu \mathrm{l}$ of the matrix solution on the $3 \mathrm{D}$ cell culture slice and allow the matrix to crystalize.

4. Alternatively, use the airbrush method. Fill a commercial grade airbrush with the matrix solution

1. Hold the sprayer 8-12 inches away from the slide, aim a fine spray at the slice. In between passes, allow the matrix to dry for 1 min to allow for the best crystal formation. Up to 10-20 passes of the airbrush is required to produce an optimal layer of matrix ${ }^{22}$.

5. Alternatively, use the sublimation method detailed elsewhere. ${ }^{25,27}$

6. Dry slides in a desiccator for at least $30 \mathrm{~min}$ prior to MALDI-MSI, regardless of the method of matrix application. ${ }^{25}$

\section{MALDI-MSI Analysis Using a MALDI-TOF System (i.e., AutoFlex III)}

NOTE: This section contains instructions and advice for setting parameters for a MALDI-TOF imaging experiment. Depending on the instrument manufacturer and software used, the process may vary.

1. Fit the slides in an adapter made to securely hold $75 \mathrm{~mm} \times 25 \mathrm{~mm}$ slides to image the 3D cell culture slices attached to ITO coated slides. Load the slide adapter into the instrument.

2. Prepare the instrument for MALDI-MSI by selecting an appropriate calibration standard for the mass range in question. For interrogation of small molecules, the signals detected that correspond to the matrix used, $\alpha-\mathrm{CHCA}$, are one common group of calibrants. For analysis of proteins, known protein standards (i.e., ubiquitin, trypsinogen) in the mass range of interest are selected and spotted adjacent to the 3D cell cultures as calibrants. Calibrate the instrument before proceeding with method development.

3. Prior to imaging, develop data acquisition methods for the mass range of choice using the software provided by the instrument manufacturer. See Figures 1 and $\mathbf{2}$ for small molecule and protein imaging. For small molecule imaging, use reflectron mode for the highest mass resolution.

1. Adjust the mass range for small molecule data acquisition between $100-1,000 \mathrm{~m} / \mathrm{z}$ and for proteins between $8,000-25,000 \mathrm{~m} / \mathrm{z}$. Adjust the mass range to encompass the region of choice while providing the highest mass resolution possible with the instrument.

2. Adjust laser power, frequency, number of laser shots and spot size using the calibration solution and a nearby 'sacrificial' 3D cell culture slice. Observe these settings in the main instrument control software. Use the following settings as a recommended starting point: $60 \%$ power, 400 laser shots, ultra small spot size. These settings will vary across instruments and methods, so optimize instrument parameters to give the highest quality spectra for each specific instrument. Other parameters that can be adjusted include the detector gain, sample rate, and electronic gain. Save this method for use in the imaging software (i.e., AutoExecute Method that FlexImaging will draw upon).

NOTE: Suppress ions below the mass range of interest (again found in the instrument control) so as to not interfere with detection.

3. Develop protein data acquisition methods for the mass range of choice. Follow the same steps outlined above, but for the mid-to-high mass ranges, above about $3 \mathrm{kDa}$, use linear mode.

NOTE: Settings will differ from those used for small molecule methods.

4. Setup specific MS instrument parameters using the imaging software provided by the instrument manufacturer, such as FlexImaging for Bruker MALDI-TOF systems.

1. Create a new imaging file and folder, using the methods developed prior and saved. Select the instrument parameters such as raster spot size (change from the default about $200 \mu \mathrm{m}$ to $50 \mu \mathrm{m}$ ), set up the instrument control method (setup in step 8.3.2 or 8.3.3), and position where to scan over the 3D cell culture.

2. Select three appropriate teach points on the sample, moving the laser to each in turn. Obtain the optical photograph from the MALDITOF laser control software using 'print screen'.

NOTE: Many imaging software programs require an optical photograph of the sample to 'teach' the software where the laser is located, which can be obtained with a scanner or often the instrument camera.

5. Then start the imaging process from the imaging software (not the instrument control) and acquire mass spectra from each slice. Observe most masses across the 3D cell culture and others localized to particular areas. 


\section{Optional Data Analysis Methods}

1. For targeted analysis, perform manual analysis of the spectra by clicking a mass in the mean spectrum, or allow the imaging software to automatically pick masses above a particular threshold (e.g., peaks above the top $20 \%$ threshold). For quality control, examine the distribution of matrix peaks or the mean spectrum.

2. Perform statistical analyses with extracted datasets in mathematical software such as $\mathrm{R}$ or Matlab.

1. Export single spectra in ASCII format, a readable text file, for loading into the software of choice.

2. Convert the individual spectra to ASCII , mzXML, of mzML format for reading by several different software programs, ${ }^{28-31}$ or export the data as an Analyze (.img) format file, a common format used for other imaging applications such as MRI. ${ }^{32}$ Two open-source options for analysis are MSiReader and BioMap ${ }^{32,33}$.

3. Once the files are exported into a format readable by the software, load the dataset via the instructions in the respective software. After loading, perform post-acquisition processing, such as baseline removal and normalization.

4. With this dataset, perform statistical treatments such as principal component analysis of hierarchical clustering using either custom scripts or built in algorithms in software such as Matlab ${ }^{18}$.

\section{Representative Results}

MSI imaging has the potential to reveal many different molecular distribution in 3D cell cultures. Using the method outlined above, species from small molecules (Figure 1) to large proteins (Figure 2) may be tracked across the culture. These results show visualization of a single ion with the free tool MSiReader. ${ }^{32}$ Results may be analyzed for single ions of interest across the 3D cell culture with visualization software either in a region of interest or the entire scanned region. Additionally most software will automatically visualize ions above a certain threshold set by the user, which may aid discovery efforts. Once single ion maps are obtained, most software includes an ability to overlay the images to view the colocalization of ions. Either in discovery-based approaches or in a targeted fashion, mass spectrometry imaging is a powerful tool to analyze 3D cell cultures.
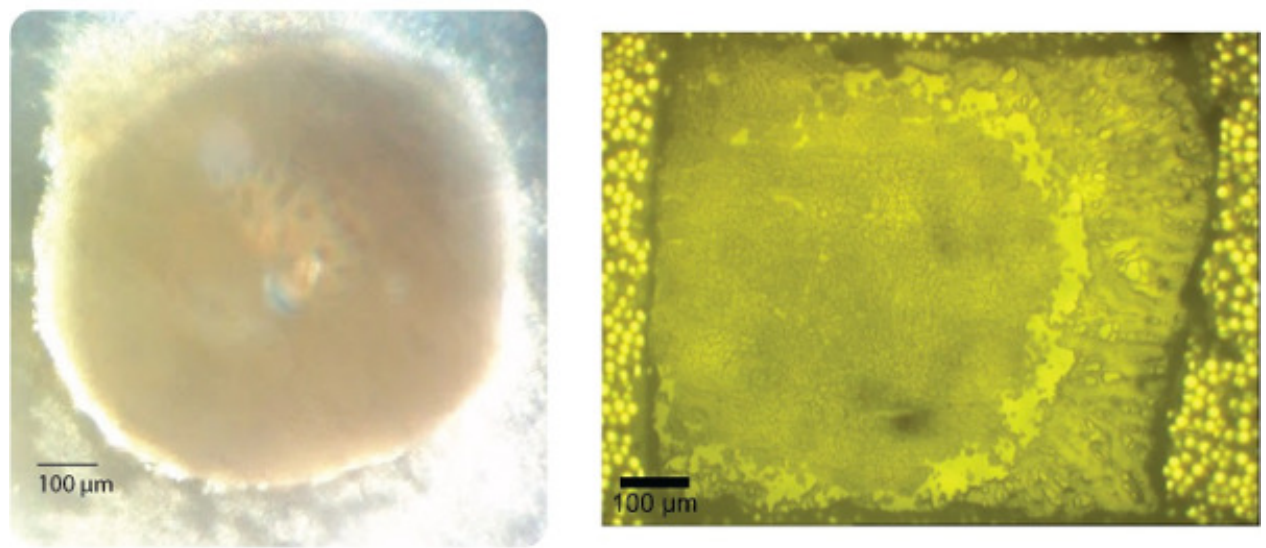

Figure 1. Representative results of 3D cell culture growth and sectioning. Left) Optical image of an intact colorectal HCT-116 3D cell culture structure as viewed on day 14 prior to harvesting. Right) Optical image of an approximately equatorial slice of a colorectal 3D cell culture thaw mounted onto an ITO-coated slide for imaging. Please click here to view a larger version of this figure. 


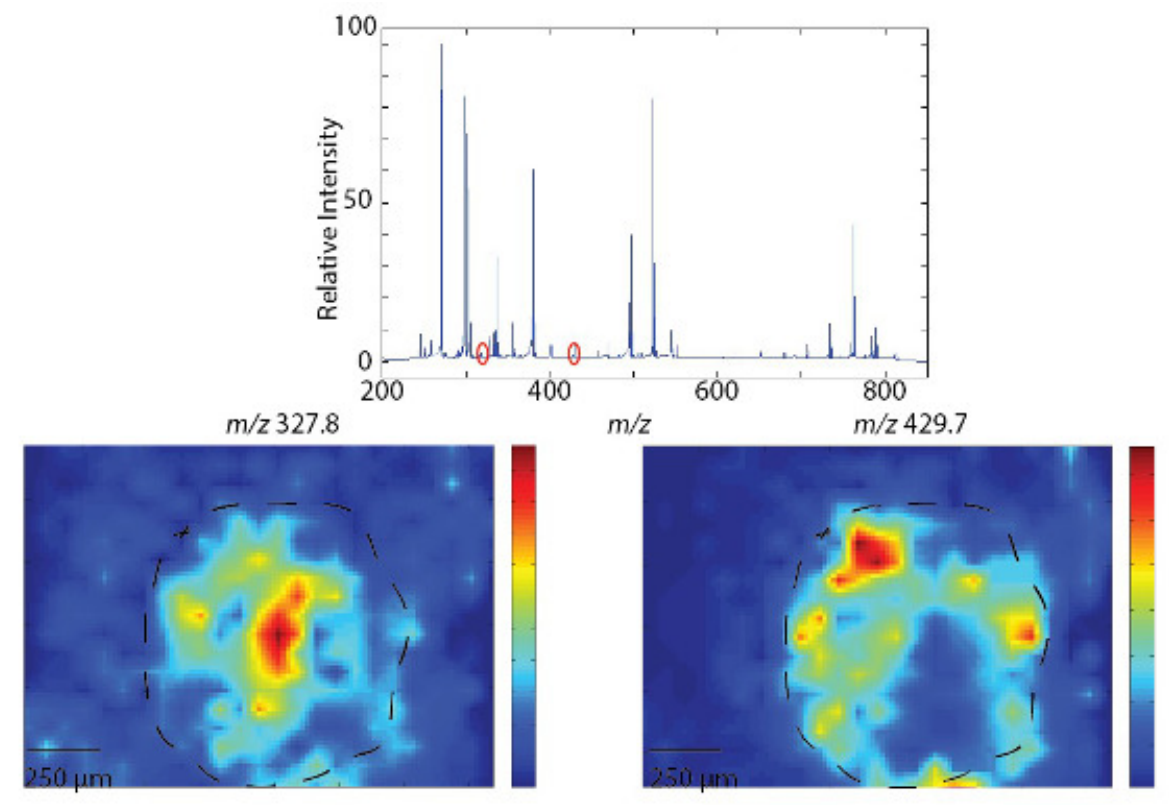

Figure 2. Representative results of small molecule MALDI-MSI of an equatorial section of a 3D cell culture. Top) Average mass spectra achieved with $\alpha-\mathrm{CHCA}$ in the low mass (small molecule) range. Bottom) Representative images of a single mass: $327.8 \mathrm{~m} / \mathrm{z}$ primarily localized to the interior of the 3D cell culture and $429.7 \mathrm{~m} / \mathrm{z}$ primarily localized to the edge of the 3D cell culture. Dashed line indicates the approximate edge of the spheroid. Please click here to view a larger version of this figure.
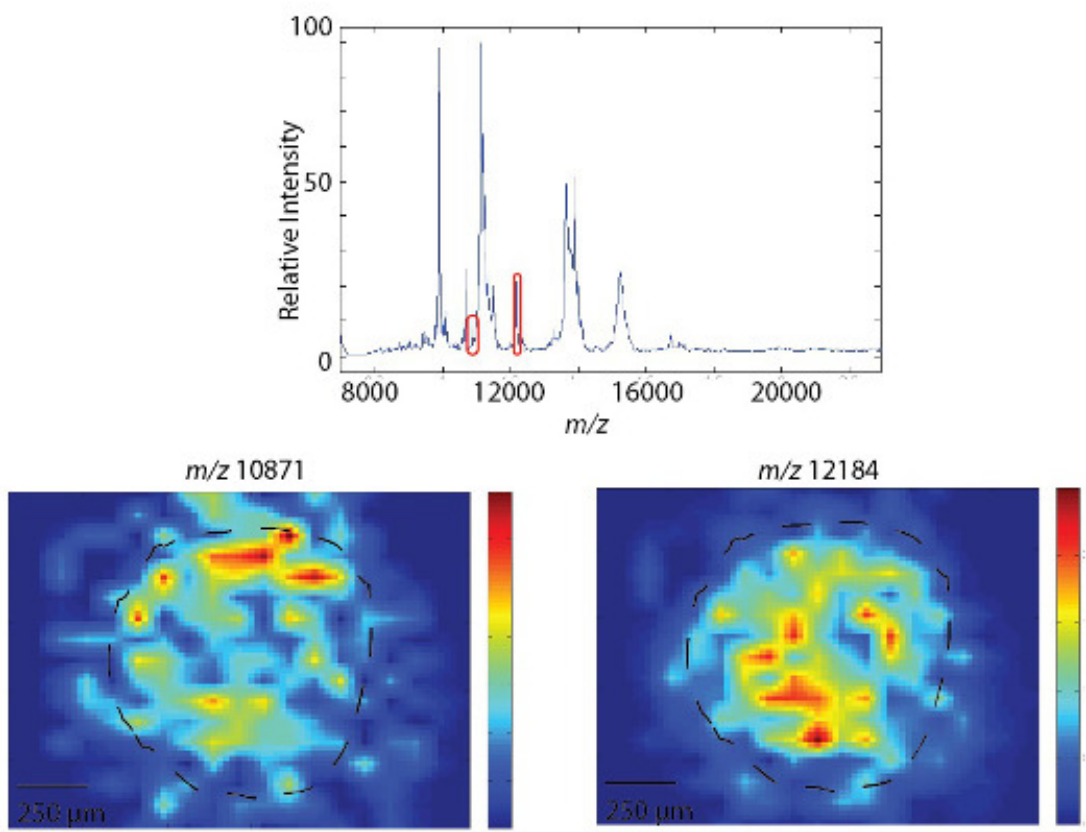

Figure 3. Representative results of protein imaging by MALDI-MSI of an equatorial section of a 3D cell culture. Top) Average mass spectra achieved with sinapic acid in the higher (protein) mass range. Bottom) Representative images of a single mass: $10,871 \mathrm{~m} / \mathrm{z}$ primarily localized to the edge of the spheroid, and $12,184 \mathrm{~m} / \mathrm{z}$ localized more toward the interior of the spheroid. Dashed line indicates the approximate edge of the spheroid. Please click here to view a larger version of this figure.

\section{Discussion}

Using the methods outlined above, 3D cell cultures may be grown and analyzed with MALDI-MSI. Many immortalized cell lines will form 3D structures when cultured appropriately. ${ }^{9,10}$ Care should be taken to cultivate cells that have not been passed too many times, that is, have low passage numbers. In general, cells with passage numbers of ten and lower are optimal for growing 3D cell cultures. Growing the 3D cell cultures in a traditional agarose support provides a cost effective way for research groups interested in 3D cell culture to try this system. This method utilizes few components not already in most mammalian cell culture laboratories. Careful attention must be paid to the preparation of the agarose plates for growth so that the $3 \mathrm{D}$ cell cultures are consistent in size and shape; some aspects that require careful attention include checking that 
no air bubbles are entrapped to the mixture and that a proper meniscus is formed at the bottom of each well. If the meniscus does not form properly, the cells may grow in non-spheroid shapes or in small clumps. Also, care must be taken not to place PBS into the gelatin with the spheroids. If this happens, remove the PBS from the top of the gelatin using a $200 \mu \mathrm{l}$ pipette. If cost is not a factor, ultra-low binding round bottom plates are commercially available and offer simplification of these steps. While other tissue embedding methods can also be costly and nonmass spectrometry compatible, gelatin-embedding has been shown to be both inexpensive and versatile. Preparation strategies outlined above have been optimized for 3D cell cultures to obtain the highest quality data. While gelatin-embedding has been shown to be compatible with mass spectrometry analysis, this process does narrow the available matrices due to co-crystallization. Once frozen, the 3D cell cultures are stable for months as long as they are not freeze-thawed. The gelatin becomes opaque when frozen, and the $3 \mathrm{D}$ cell cultures are of a similar color, so it is advisable before freezing to document the 3D cell culture placement to aid in slicing.

When preparing slides, matrix can be applied with several different methods, but it should be noted that some matrixes have been found to cocrystalize with the gelatin, such as ferulic acid, rendering the sample unusable. Smaller matrix crystals produce more consistent mass spectra. While handspotting is the simplest method of matrix application, the larger solvent volume can result in larger crystal sizes and significant analyte migration.

At the mass spectrometer, advances in MALDI-MSI technologies have reduced the expertise required for beginning analyses. Data acquisition and analysis is straightforward on most systems, allowing even a novice to obtain high quality information from ITO-coated slides. Careful selection of the instrument parameters, optimized on nearby non-image worthy 3D cell cultures, is critical to obtaining high quality data. For instance, increasing laser power may increase peak intensity but decreasing laser power may improve resolution and give more symmetrical peak shapes. 3D cell cultures should be used quickly after slicing to ensure optimal sample quality. Degradation of protein signal may be seen in less than a week without proper storage (dessicator/- $80^{\circ} \mathrm{C}$ freezer), particularly in the high mass region (above $\left.20 \mathrm{kDa}\right)$. Due to growing demand, many different visualization software programs have been developed and are free to the research public. Most imaging mass spectrometers have installed the software necessary to visualize single masses with the proprietary formats used on each instrument. These software programs may be used to obtain single-mass images and export the data. Most software will also allow the overlay of two or more masses of interest. Additionally, many different viewers for MSI data are free to download, such as MSiReader and BioMap. ${ }^{32,33}$ The mass spectrometry data obtained may also be processed post-acquisition with ease, bringing more useful information to the forefront.

From pharmaceuticals to lipids to proteins, the system outlined above allows for rapid acquisition of data to evaluate the distribution of molecules of interest across a 3D cell culture structure. Serial sections can be taken to image the entire 3D structure by building from each 2D image of a slice of the 3D cell culture. The techniques and notes in the protocol will be of use to researchers wishing to begin three-dimensional cell culture as a bridge between monolayer culture and animal models. Once mastered, these techniques can be applied to multiple types of 3D cell cultures, tissues, and cell cultures, allowing researchers to image whichever samples they choose.

\section{Disclosures}

This article is part of a special issue on Multimodal Pre-Clinical Imaging, sponsored by Bruker Biospin. The MALDI-TOF system used to obtain the representative results is a Bruker AutoFlex III.

\section{Acknowledgements}

This work was supported by the Walther Cancer Foundation under the Advancing Basic Cancer Research program to the Harper Cancer Research Institute of the University of Notre Dame (DARW) and the 2011 Starter Grant from the Society for Analytical Chemists of Pittsburgh $(\mathrm{ABH})$. Thanks also to the staff of the Mass Spectrometry and Proteomics Facility for useful discussions.

\section{References}

1. Schober, Y., Guenther, S., Spengler, B., \& Römpp, A. Single cell matrix-assisted laser desorption/ionization mass spectrometry imaging. Anal Chem. 84 (15), 6293-7, doi:10.1021/ac301337h, (2012).

2. Cornett, D., Frappier, S., \& Caprioli, R. MALDI-FTICR imaging mass spectrometry of drugs and metabolites in tissue. Anal Chem. 80 (14), 5648-5653, doi:10.1021/ac800617s.MALDI-FTICR, (2008).

3. Reyzer, M. L., Hsieh, Y., Ng, K., Korfmacher, W. a \& Caprioli, R. M. Direct analysis of drug candidates in tissue by matrix-assisted laser desorption/ionization mass spectrometry. J Mass Spectrom. 38 (10), 1081-92, doi:10.1002/jms.525, (2003).

4. Reyzer, M. L., Chaurand, P., Angel, P. M., \& Caprioli, R. M. Direct molecular analysis of whole-body animal tissue sections by MALDI imaging mass spectrometry. Methods Mol Biol. 656 (18), 285-301, doi:10.1007/978-1-60761-746-4_17, (2010).

5. Cornett, D., Reyzer, M., Chaurand, P., \& Caprioli, R. MALDI imaging mass spectrometry: molecular snapshots of biochemical systems. Nat Methods. 4 (10), 828-833, doi:10.1038/NMETH1094, (2007).

6. Guenther, S., et al. Histology by mass spectrometry: label-free tissue characterization obtained from high-accuracy bioanalytical imaging. Angew Chem Int Ed Engl. 49 (22), 3834-8, doi:10.1002/anie.200905559, (2010).

7. Schwamborn, K., \& Caprioli, R. M. Molecular imaging by mass spectrometry--looking beyond classical histology. Nat Rev Cancer. 10 (9), 639-46, doi:10.1038/nrc2917, (2010).

8. Kunz-Schughart, L. a, Kreutz, M., \& Knuechel, R. Multicellular spheroids: a three-dimensional in vitro culture system to study tumour biology. Int J Exp Pathol. 79 (1), 1-23 doi: 10.1046/j.1365-2613.1998.00051.x, (1998).

9. Hirschhaeuser, F., Menne, H., Dittfeld, C., West, J., Mueller-Klieser, W., \& Kunz-Schughart, L. a Multicellular tumor spheroids: an underestimated tool is catching up again.J Biotechnol. 148 (1), 3-15, doi:10.1016/j.jbiotec.2010.01.012, (2010).

10. Friedrich, J., Seidel, C., Ebner, R., \& Kunz-Schughart, L. A. Spheroid-based drug screen: considerations and practical approach. Nat Protoc. 4 (3), 309-24, doi:10.1038/nprot.2008.226, (2009). 
11. Lee, G. Y., Kenny, P. A., Lee, E. H., \& Bissell, M. J. Three-dimensional culture models of normal and malignant breast epithelial cells. Nat Methods. 4 (4), 359-365, doi:10.1038/NMETH1015, (2007).

12. Yamada, K. M., \& Cukierman, E. Modeling tissue morphogenesis and cancer in 3D. Cell. 130 (4), 601-10, doi:10.1016/j.cell.2007.08.006, (2007).

13. Fernandes, T. G., Kwon, S.-J., et al. Three-dimensional cell culture microarray for high-throughput studies of stem cell fate. Biotechnol Bioeng. 106 (1), 106-18, doi:10.1002/bit.22661, (2010).

14. Meli, L., Jordan, E. T., Clark, D. S., Linhardt, R. J., \& Dordick, J. S. Influence of a three-dimensional, microarray environment on human cell culture in drug screening systems. Biomaterials. 33 (35), 9087-96, doi:10.1016/j.biomaterials.2012.08.065, (2012).

15. Weaver, E. M., \& Hummon, A. B. Imaging mass spectrometry: From tissue sections to cell cultures. Adv Drug Deliv Rev. 65 (8), $1039-55$, doi:10.1016/j.addr.2013.03.006, (2013).

16. Li, H., \& Hummon, A. B. Imaging mass spectrometry of three-dimensional cell culture systems. Anal Chem. 83 (22), 8794-801, doi:10.1021/ ac202356g, (2011).

17. Liu, X., Weaver, E. M., \& Hummon, A. B. Evaluation of Therapeutics in Three-Dimensional Cell Culture Systems by MALDI Imaging Mass Spectrometry. Anal Chem. 85 (13), 6295-302, doi:10.1021/ac400519c, (2013).

18. Ahlf, D. R., Masyuko, R. N., Hummon, A. B., \& Bohn, P. W. Correlated Mass Spectrometry Imaging and Confocal Raman Microscopy for Studies of Three-Dimensional Cell Culture Sections. Submitted. (2014).

19. Derda, R., Tang, S. K. Y., et al. Multizone paper platform for 3D cell cultures. PloS one. 6 (5), e18940, doi:10.1371/journal.pone.0018940. (2011).

20. Imbeault, S., Valenzuela, N., Fai, S., \& Bennett, S. a L. Localizing protein in 3D neural stem cell culture: a hybrid visualization methodology. J Vis Exp. 2 (46), 2-7, doi:10.3791/2483, (2010).

21. He, W., Kuang, Y., et al. Proteomic Comparison of 3D and 2D Glioma Models Reveals Increased HLA-E Expression in 3D Models is Associated with Resistance to NK Cell-Mediated Cytotoxicity. J Proteome Res. 13 (5), 2272-2281, doi:10.1021/pr500064m, (2014).

22. Chen, R., Cape, S. S., Sturm, R. M., \& Li, L. Mass Spectrometric Imaging of Neuropeptides in Decapod Crustacean Neuronal Tissues. Methods Mol Biol. 656, 451-463, doi:10.1007/978-1-60761-746-4, (2010).

23. DeKeyser, S. S., Kutz-Naber, K. K., Schmidt, J. J., Barrett-Wilt, G. A., \& Li, L. Imaging Mass Spectrometry of Neuropeptides in Decapod Crustacean Neuronal Tissues. J Proteome Res. 6 (5), 1782-1791, doi:10.1021/pr060603v, (2007).

24. Van Hove, E. R. A., Smith, D. F., Fornai, L., Glunde, K., \& Heeren, R. M. a An alternative paper based tissue washing method for mass spectrometry imaging: localized washing and fragile tissue analysis. J Am Soc Mass Spectrom. 22 (10), 1885-90, doi:10.1007/ s13361-011-0203-z, (2011).

25. Yang, J., \& Caprioli, R. M. Matrix Sublimation/Recrystalization for Imaging Proteins by Mass Spectrometry at High Spatial Resolution. Anal Chem. 83 (14), 5728-5734, doi:10.1021/ac200998a.Matrix, (2012).

26. Seeley, E. H., Oppenheimer, S. R., Mi, D., Chaurand, P., \& Caprioli, R. M. Enhancement of protein sensitivity for MALDI imaging mass spectrometry after chemical treatment of tissue sections. J Am Soc Mass Spectrom. 19 (8), 1069-77, doi:10.1016/j.jasms.2008.03.016, (2008).

27. Gemperline, E., \& Li, L. MALDI-mass spectrometric imaging for the investigation of metabolites in Medicago truncatula root nodules. $J$ Vis Exp.(85), 1-11, doi:10.3791/51434, (2014).

28. Deutsch, E. mzML: a single, unifying data format for mass spectrometer output. Proteomics. 8 (14), 2776-2777, doi: 10.1002/ pmic.200890049, (2008).

29. Pedrioli, P. G. a, Eng, J. K., et al. A common open representation of mass spectrometry data and its application to proteomics research. Nat Biotechnol. 22 (11), 1459-66, doi:10.1038/nbt1031, (2004).

30. Lin, S., Zhu, L., Winter, A., Sasinowski, M., \& Kibbe, W. What is mzXML good for? Expert Rev Proteomics. 2 (6), 839-845, doi:10.1586/14789450.2.6.839, (2005).

31. Orchard, S., Hoogland, C., Bairoch, A., Eisenacher, M., Kraus, H.-J., \& Binz, P.-A. Managing the data explosion. A report on the HUPO-PSI Workshop. August 2008, Amsterdam, The Netherlands. Proteomics. 9 (3), 499-501, doi:10.1002/pmic.200800838, (2009).

32. Robichaud, G., Garrard, K. P., Barry, J. a \& Muddiman, D. C. MSiReader: an open-source interface to view and analyze high resolving power MS imaging files on Matlab platform. J Am Soc Mass Spectrom. 24 (5), 718-21, doi:10.1007/s13361-013-0607-z, (2013).

33. Andersson, M., Groseclose, M. R., Deutch, A. Y., \& Caprioli, R. M. Imaging mass spectrometry of proteins and peptides : 3D volume reconstruction. Nat Methods. 5 (1), 101-108, doi:10.1038/NMETH1145, (2008). 